\title{
Estilos de vida de los estudiantes universitarios: una revisión sistemática
}

\author{
Lifestyles of university students: a systematic review \\ Estilos de vida dos estudantes universitários: uma revisão sistemática
Marlucio De Souza Martins ${ }^{1}$; Melba Ximena Figueroa-Ángel ${ }^{2}$ Pontificia Universidad Javeriana, PUJ, Bogotá, Colombia
UNIVERSIDAd SANTO TOMás, USTA, BOGOTÁ, COLOMBIA

\begin{abstract}
RESUMEN
Este trabajo tiene como objetivo presentar una revisión sistemática documental para el desarrollo de un marco de referencia sobre los estilos de vida en el contexto universitario actual en relación con la calidad de vida. Los estilos de vida representan acciones cotidianas que reflejan actitudes y valores de las personas, que están relacionados directamente con su calidad de vida. Se utilizó un método cualitativo descriptivo a través de una revisión sistemática documental. Además, como fuente de información se utilizaron trabajos publicados en las bases de datos Redalyc, Scopus, Elsevier, Ebsco y SpringerLink durante el período 2014 al 2020. Para el análisis descriptivo de la información se seleccionaron 47 documentos que cumplían con los criterios de selección. Los resultados muestran una tendencia de conducta de estilos de vida no saludables en los estudiantes universitarios, lo que a futuro puede generar un deterioro de la salud y calidad de vida.
\end{abstract}

Palabras clave: Estilo de Vida. Calidad de Vida. Estudiantes Universitarios. Revisión Sistemática.

\begin{abstract}
The objective of this work is to present a systematic documentary review for the development of a frame of reference on lifestyles in the current university context in relation to quality of life. Lifestyles represent everyday actions that reflect people's attitudes and values, which are directly related to their quality of life. A descriptive qualitative method was used, through a systematic documentary review. Furthermore, as a source of information, works published in the Redalyc, Scopus, Elsevier, Ebsco and SpringerLink databases were used during the period 2014 to 2020. For the descriptive analysis of the information, 47 documents were selected that met the selection criteria. The results show a trend of unhealthy lifestyle behavior in university students, which in the future can lead to a deterioration in health and quality of life.
\end{abstract}

Keywords: Lifestyle. Quality of Life. University Students. Systematic Review.

\section{RESUMO}

Este trabalho tem como objetivo apresentar uma revisão sistemática documental para o desenvolvimento de um marco referencial sobre os estilos de vida no atual contexto universitário, atentando-se à qualidade de vida. Os estilos de vida representam ações cotidianas que refletem as atitudes e valores das pessoas, que estão diretamente relacionados à sua qualidade de vida. Foi utilizado um método descritivo qualitativo, por meio de uma revisão documental sistemática. Além disso, foram utilizados trabalhos publicados nas bases de dados Redalyc, Scopus, Elsevier, Ebsco e SpringerLink no período de 2014 a 2020. Para a análise descritiva da informação, foram selecionados 47 documentos que atenderam aos critérios de seleção. Os resultados mostram uma tendência de comportamentos de estilo de vida não saudáveis em estudantes universitários, que no futuro podem levar a uma deterioração da saúde e da qualidade de vida.

Palavras-chave: Estilo de vida. Qualidade de Vida. Estudantes Universitários. Revisão Sistemática.

\footnotetext{
${ }^{1}$ Docente Investigador del Departamento de Formación de la Facultad de Educación de la PUJ. E-mail: mdesouzamartins@javeriana.edu.co. ORCID: http://orcid.org/0000-0001-8137-6891.

${ }^{2}$ Docente Investigadora del Doctorado en Psicología de la USTA. E-mail: melbafigueroa@usantotomas.edu.co. ORCID: http://orcid.org/0000-0003-0420-1590.
} 


\section{INTRODUCCIÓN}

El ser humano desde su existencia se encuentra en un constante proceso de evolución y cambios de comportamientos que están asociados con el entorno a partir del contexto social y cultural. En este sentido, está influenciado por los elementos naturales, sociales y culturales desde su nacimiento, ya que, de acuerdo con Galindo (2016, p. 147) "[...] el ser humano es un ser biopsicosocial inmerso en interacción constante con su entorno". De allí, que los aspectos biológicos, psicológicos y sociales influyen en el desarrollo, comportamiento y bienestar del ser humano, apoyando la configuración de estilos de vida que dan cuenta de esa relación.

En esa misma línea, Bronfenbrenner (1987) plantea que el comportamiento humano está asociado al entorno a partir de diferentes estructuras y niveles de este, lo que determina las conductas e influye en el estilo de vida del ser humano.

Así mismo, estos aspectos, a lo largo de los siglos están asociados a los avances y creaciones tecnológicas derivadas de las necesidades y de las innovaciones humanas, para facilitar y beneficiar el trabajo diario de las personas. Según Carrillo (2017, p. 269) “[...] el mundo atraviesa cambios económicos, sociales, culturales, políticos y tecnológicos con una rapidez impresionante debidos principalmente a la revolución industrial, tecnológica y globalización que dinamizan la interacción mundial en todo aspecto".

Por lo anterior, la relación del comportamiento humano con el entorno y el contexto en que la persona vive, de acuerdo con la Organización Mundial de la Salud WHO (2000), define el estilo de vida del ser humano, ya que presenta interacciones entre las condiciones de vida y patrones de conductas individuales que son determinados por las características biopsicosociales y por el contexto social y cultural. Del mismo modo, González-Páramo (2017) y Carrillo (2017), afirman que el comportamiento y desarrollo humano a partir de la Revolución Industrial está asociado al alcance de un bienestar físico y psicológico, y de estilo de vida, que están relacionados con el progreso económico, social y cultural de las personas.

Para Pew Research Center (2010), los efectos de la Revolución Industrial inciden en las nuevas generaciones, en donde la edad más afectada por este cambio se encuentra entre el rango de los 18 y 29 años (edad de la etapa denominada - vida universitaria), porque "[...] todavía los jóvenes adultos están desarrollando sus valores fundamentales y estos eventos permanecen con ellos a medida que avanzan en su ciclo vital" (p. 4). En este sentido, la generación que más se encuentra influida por los avances tecnológicos, conforme Álvarez y Haro (2017), son los 'Millennials Generation' (nacidos entre 1980 y 1996) y los 'Centennial Generation' (nacidos entre 1997 y 2016), que, debido a un "[...] proceso de modernización, globalización y desarrollo tecnológico de la humanidad, reflejan nuevas conductas y estilos de vida en la sociedad" (DE SOUZA MARTINS et al. 2017, p. 232).

La preocupación por el estilo de vida surge a partir de las investigaciones dadas desde el siglo XX y principios del siglo XXI, las cuales afirman que el estilo de vida es un esquema de comportamiento que comprende hábitos y que se reflejan en el bienestar psicológico de las personas. Nahas (2013), afirma que el estilo de vida es comprendido como el conjunto de acciones habituales que reflejan las actitudes los valores y las oportunidades de vida de las personas, además, está constituido por cinco factores principales como: el estrés, la nutrición, las relaciones sociales, el comportamiento preventivo y la actividad física.

A su vez, Nahas (2013), considera que la nutrición, el estrés, la actividad física, el comportamiento preventivo y las relaciones sociales, son los componentes que afectan 
la salud de las personas y están asociados al desarrollo de las Enfermedades Crónicas No Transmisibles - ECNT.

Hay que mencionar que el estilo de vida es un comportamiento del ser humano que sufre transformaciones desde lo biopsicosocial, lo que puede desarrollar cambios de actitudes y comportamientos para satisfacer las necesidades dentro del desarrollo personal del individuo (WHO, 2000). En concordancia con lo anterior, los seres humanos en sus " [...] diferentes etapas, considerando la variabilidad e individualidad de cada persona, establecen su propio estilo de vida, el cual está íntimamente relacionado con la salud" (COTRINA; RODRÍGUEZ, 2014, p. 20).

Para De Souza Martins, Molina Pinzon y Zea Robles (2015), los estilos de vida representan acciones cotidianas que reflejan actitudes y valores de las personas que están relacionados directamente con su calidad de vida. Gómez-Acosta (2018), menciona que los estilos de vida son concebidos como un conjunto de conductas que determinan el proceso de salud-enfermedad en combinación con la vulnerabilidad biológica, la reactividad psicofisiológica, la edad y el sexo.

Lo dicho hasta aquí, supone que existe una conexión entre desarrollo humano, ciclo vital y estilo de vida, que está relacionada con la salud y calidad de vida de las personas, en particular, en los jóvenes universitarios, ya que el inicio de la vida universitaria es la etapa donde se presentan los cambios de comportamientos que pueden afectar su estilo de vida y calidad de vida (PAPALIA; FELDMAN; MARTORELL, 2012; HARRIS, 2019).

De acuerdo con Papalia, Feldman y Martorell (2012), la universidad es un entorno en que los jóvenes universitarios inician comportamientos y un estilo de vida no saludables, ya que se encuentran inmersos en un contexto propicio para el desarrollo de hábitos no favorables de consumo (cigarrillo, alcohol), de alimenticios (comidas rápidas e industrializadas), y de actividad física (bajo grado e irregular) que producen cambios graduales en el organismo y pueden afectar la salud.

Bravo-Avalos et al. (2020), destacan el aumento del consumo de alimentos industrializados entre los estudiantes universitarios como "[...] hamburguesas, salchipapas, papas fritas, pollo frito, chocolates golosinas, pizza, entre otras, que son de fácil consumo por su portabilidad" (p. 97). Además, el consumo de este tipo de alimentos está relacionado con enfermedades como el sobrepeso, obesidad, diabetes, problemas cardiovasculares, presión alta y también se elevan los niveles de colesterol y triglicéridos; además manifiesta que este consumo es más visible en el periodo de la universidad ya que "[...] la falta de tiempo y un cambio en los hábitos alimenticios hace que las personas opten por consumir estos alimentos" (BRAVO-AVALOS et al. 2020, p. 97).

Este aspecto es de especial interés, ya que de acuerdo con Alonso, Martínez y Banda (2019), la nutrición constituye uno de los factores con mayor influencia en el estilo de vida y la salud de los jóvenes universitarios, ya que al "[...] realizar una dieta suficiente, equilibrada y adaptada a las necesidades en las diferentes etapas de la vida, garantiza un adecuado crecimiento físico, facilita el desarrollo psicológico, ayuda en la prevención de enfermedades" (p. 85).

Por otro lado, Quiliano y Quiliano (2020 p. 2), afirman que los niveles de exigencia en el ámbito académico universitario " [...] por lo general está acompañado de estrés académico, a causa de un inadecuado desarrollo de habilidades que estimula la inteligencia emocional". En este sentido, el control del estrés es un factor muy importante para el desarrollo humano y está asociado a un estilo de vida.

Para Acosta, Jiménez y Barba (2019), los jóvenes se caracterizan por estar en una etapa de la vida evolutiva "[...] física-psicológica de gran interés, y a su vez, inmersa en 
un entorno sociocultural cambiante como es el paso de la secundaria a la universidad, superar la mayoría de edad y un estilo de vida abierto a nuevas experiencias" (p. 33). En este sentido, la actividad física es un factor importante en el desarrollo de un estilo de vida saludable. Da Costa et al. (2019), han mostrado que solamente el 12\% de los estudiantes dedican tiempo para realizar actividad física moderada-a-vigorosa y $41 \%$ en actividad física ligera, así, el restante $47 \%$ se emplea en actividades que no implican movimiento, lo que demuestra un estilo de vida no saludable en la población.

En esa misma línea de complejidad, De Souza Martins et al. (2017), evidenciaron un índice negativo en los estilos de vida en el contexto universitario, que el "[...] 47\% de los estudiantes son considerados sedentarios y no realizan actividad física de forma moderada [...], el 35\% no realiza ninguna actividad que involucre ejercicios de fuerza a la semana" (p. 237). Esta conducta, afecta la salud y calidad de vida de los jóvenes universitarios a partir de un estilo de vida no saludable en que la inactividad física causa el desarrollo de las principales ECNT.

Por otro lado, Rojas Ospina et al. (2018), plantean que en el contexto universitario los estudiantes se enfrentan a múltiples cargas y responsabilidades asociadas con las relaciones sociales, lo cual, repercute en el estilo de vida de los universitarios de manera positiva o negativa en aspectos como la salud y las relaciones interpersonales, ya que, de acuerdo con Papalia, Feldman y Martorell (2012), las relaciones sociales en el contexto universitario, derivadas de las reuniones y encuentros entre pares, pueden aumentar los hábitos y estilos de vida no favorables de consumo de cigarrillo, alcohol y sustancias psicoactivas, lo que puede generar un deterioro de la salud y de la calidad de vida.

Para De Souza Martins et al. (2017) y el American College of Sports Medicine ACSM (2018), el crecimiento de un estilo de vida negativo en la sociedad trae como consecuencia la aparición de ECNT, como: hipertensión arterial, diabetes mellitus II y obesidad. Entre las ECNT más frecuentes se encuentran las cardiovasculares, las cerebrovasculares, el cáncer, la diabetes y otros trastornos metabólicos, las enfermedades respiratorias y pulmonares, los trastornos osteomioarticulares y las enfermedades renales (ACSM, 2018; GRAU, 2016).

Estudios indican que más de 57 millones de muertes de la población mundial fueron causadas por ECNT, que comprende principalmente, enfermedades cardiovasculares $(48 \%)$, cáncer $(21 \%)$, enfermedades respiratorias crónicas (12\%) y diabetes (3,5\%) (WHO, 2013; ARESTEDT; PERSSON; BENZEIN, 2014).

Según Krupp y Chatton (2014), el consumo de tabaco es el primer factor de decesos en América Latina, seguido por la inactividad física, que aporta el $28 \%$ de las muertes relacionadas con ECNT. Otros estudios realizados por Vidarte-Claros, VélezÁlvarez y Parra-Sánchez (2012), determinan que el nivel de sedentarismo tiene una prevalencia del $73 \%$ en la población general colombiana; por género, el nivel de sedentarismo en hombres atiende al $61 \%$ y en mujeres al $84 \%$.

Ahora bien, dentro de esta comprensión del estilo de vida, la conducta preventiva puede favorecer el desarrollo del ser humano. De acuerdo con la WHO (2013), la ausencia del autocuidado y hábitos preventivos pueden relacionarse con las muertes por accidentes vitales, principalmente ocasionados por jóvenes que se encuentran bajo la influencia de sustancias psicoactivas (depresoras, estimulantes, psicodélicas), que son la primera causa de la mortalidad en esta etapa de la vida, seguida por las enfermedades de transmisión sexual y por último el suicidio.

Para el Centers for Disease Control and Prevention (2018), un estilo de vida saludable está asociado con la mejoría de la calidad de vida, que está compuesta del bienestar físico, mental y social, donde se vincula la condición para satisfacer las 
necesidades humanas (materiales, psicológicas, sociales y del entorno). En este sentido, a partir del panorama presentado, se presenta una revisión sistemática documental para el desarrollo de un marco de referencia sobre los estilos de vida con relación a las aproximaciones conceptuales y metodológicas de la calidad de vida en el contexto universitario actual.

\section{MÉTODO}

El enfoque metodológico utilizado para este trabajo está centrado en un estudio cualitativo descriptivo, a través de una revisión sistemática documental para el desarrollo de un marco de referencia sobre los estilos de vida en el contexto universitario actual en relación con calidad de vida. De acuerdo con Hernández Sampieri, Fernández Collado y Baptista Lucio (2014, p. 92), este tipo de metodología busca "[...] especificar propiedades y características importantes de cualquier fenómeno que se analice, además de describir las tendencias y particularidades de un grupo o población".

Por lo anterior, una de las fases del proceso de investigación cualitativa está centrada en la realización de una revisión de la literatura, desde la perspectiva del fenómeno y componentes del estudio, soportado en una holística teórica de revisión sistemática que "[...] implica exponer y analizar las teorías, las conceptualizaciones, las investigaciones previas y los antecedentes en general que se consideren válidos para encuadrar el estudio" (HERNÁNDEZ SAMPIERI; FERNÁNDEZ COLLADO; BAPTISTA LUCIO, 2014, p. 60).

A su vez, la revisión sistemática está centrada en identificar los planteamientos, conceptos, variables y definiciones, muestras, instrumentos y análisis realizados en otras investigaciones. Para Aguilera-Eguía (2014, p. 359), “[...] las revisiones sistemáticas son una forma de investigación que recopila y proporciona un resumen sobre un tema específico" a través de un método organizado que orientan tanto a la descripción, y explicación de la realidad, así como a la previsión de los fenómenos observados.

Para la revisión sistemática de este trabajo se optó por utilizar las etapas propuestas por Khan et al. (2003) y Moher et al. (2009), a través de la búsqueda, identificación, selección, elección e interpretación de las investigaciones para la realización de un análisis y reflexión sobre el fenómeno, conforme se presenta en la Figura 1. 
Figura 1: Fases de la Revisión Sistemática.

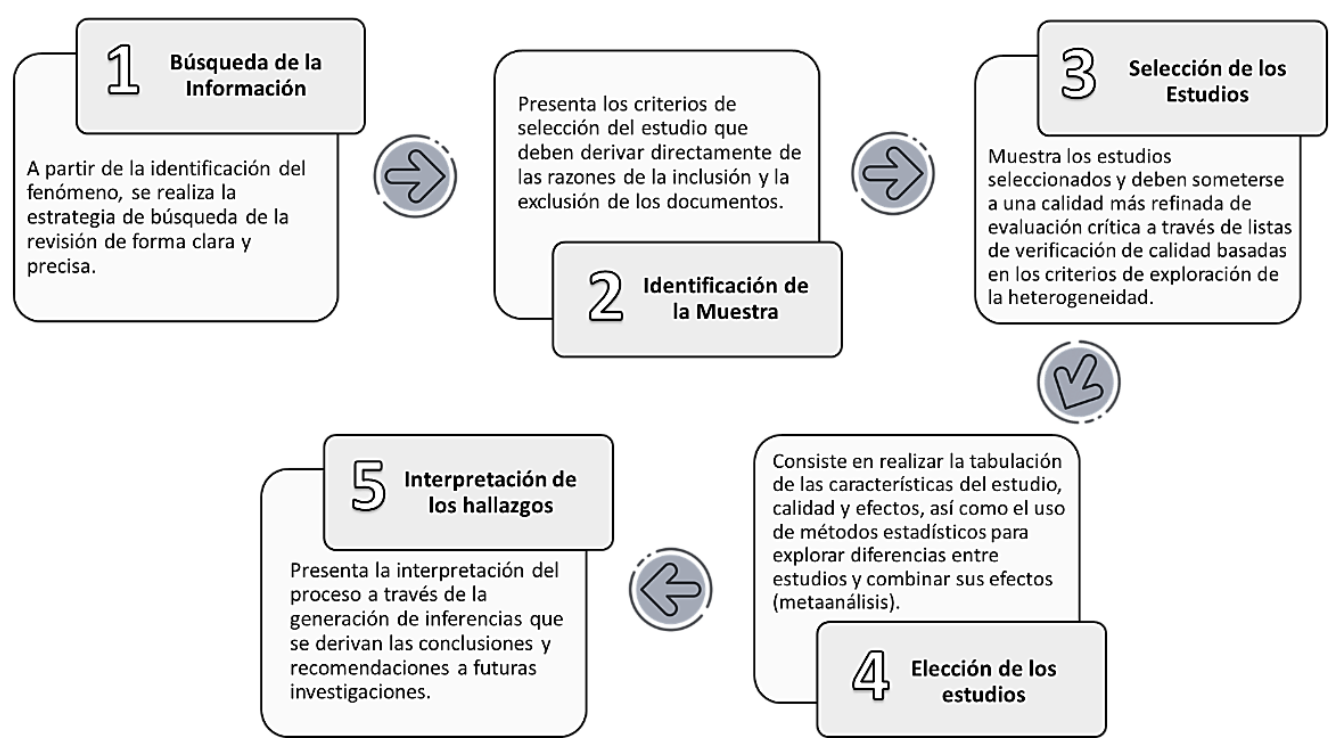

Fuente: Adaptado de Khan et al. (2003); Moher et al. (2009).

\section{BÚSQUEDA DE LA INFORMACIÓN}

En esta primera fase, el procedimiento de búsqueda se inicia a partir de las categorías de Estilos de Vida relacionados con la Calidad de Vida en Estudiantes Universitarios. Como fuente de información, se utilizaron trabajos publicados en las bases de datos Redalyc, Scopus, Elsevier, Ebsco y SpringerLink durante el período comprendido entre enero de 2014 y abril de 2020. Las palabras clave empleadas en la búsqueda fueron: «Estilos de Vida» y «Lifestyle», combinadas a través de conectores como AND/OR con: «Salud» y «Health», «Calidad de Vida» y «Quality of Life», «Bienestar»y «Wellbeing», «Estudiantes Universitarios»y «University Students».

\section{IDENTIFICACIÓN DE LA MUESTRA}

Los criterios de inclusión de referencias bibliográficas para la presente revisión sistemática fueron: 1) artículos publicados en revistas científicas de educación y salud en el periodo comprendido entre enero de 2014 y abril de 2020;2) artículos con las palabras clave seleccionadas.; 3) estudios realizados en estudiantes universitarios; y 4) publicaciones en español, portugués o inglés. A su vez, los criterios de exclusión de la revisión fueron: 1) artículos anteriores al año 2014; 2) investigaciones o artículos realizados desde otras áreas del conocimiento como: antropología, filosofía y teología; 3) artículos duplicados y 4) artículos que no fueran publicados en revistas científicas indexadas.

\section{SELECCIÓN DE LOS ESTUDIOS}

En la tercera fase, a través de los boleadores de búsqueda, se encontraron un total de 45,391 trabajos en 94 revistas registradas en las bases de datos. A su vez, se aplicaron los criterios de inclusión y exclusión. En este sentido, se eliminaron: 24,523 trabajos fuera del período de selección, 735 estudios duplicados, y 18.410 artículos de otras disciplinas. Por lo anterior, fueron seleccionados 1,723 trabajos para lectura de título y resumen. Posterior a ello, se eligieron 328 documentos para la lectura de los textos completos y, de estos, 281 artículos fueron excluidos por presentar resultados parciales y empíricos en las investigaciones, para responder a los criterios de la fase 
tres de Khan (2003) y Moher (2009). Siendo así, después de la última depuración, quedaron un total de 47 trabajos incluidos en la elección para estudio y análisis. En la Figura 2, se presentan las etapas de la revisión sistemática de los estilos de vida de los estudiantes universitarios.

Figura 2: Selección de los estudios.

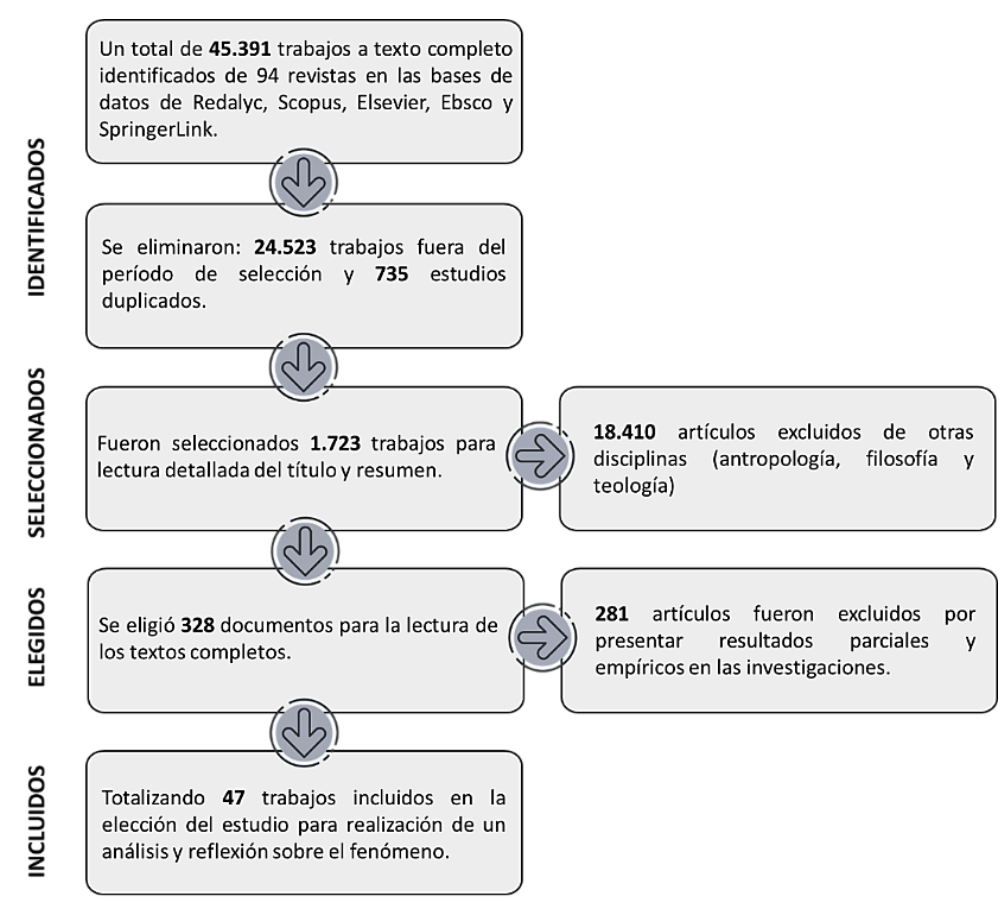

Fuente: Elaboración propia.

\section{ELECCIÓN DE LOS ESTUDIOS}

En la cuarta fase, a partir de las etapas anteriores del proceso de revisión sistemática, se seleccionaron 47 documentos para lectura a texto completo. Posteriormente, se organizó una base de datos en el programa Excel para su análisis. En este sentido, para cada artículo se registró la siguiente información: año de publicación, autores, revista de publicación, idioma, título, resumen, palabras clave, objetivos, metodología, instrumentos y principales resultados.

\section{INTERPRETACIÓN DE LOS HALLAZGOS}

En esta última fase, para realizar la interpretación de los resultados, se utilizó un análisis de revisión descriptiva a partir de la metodología, los instrumentos y los resultados derivados de los documentos seleccionados. Según Bloomberg y Volpe (2016), la revisión descriptiva sistemática de la literatura proporciona un panorama equilibrado de los principales conceptos, teorías y datos actuales del fenómeno con aporte para el conocimiento actual, además de propiciar el pensamiento crítico sobre la temática. También, se utilizó un análisis lexicométrico como parámetro para el agrupamiento de categorías, donde se extraen los principales factores de estilo de vida hallados en los artículos seleccionados a partir de cuatro dimensiones: Actividad Física, Salud, Frecuencia de Consumo y Psicológico; las cuáles, se obtienen de la agrupación de los términos en categorías que los incluyen y permiten su revisión holística. 


\section{RESULTADOS Y DISCUSIÓN}

Los resultados evidencian un aumento de investigaciones y publicaciones sobre estilo de vida en el contexto universitario en los últimos años; el 23\% de los trabajos se encuentran entre los años de 2014 y 2015, el 17\% entre los años de 2016 y 2017 y el $60 \%$ entre los años de 2018 y 2020; demostrando que sigue siendo una temática relevante en el ámbito educativo superior, y a su vez, reconoce fenómenos y problemáticas asociadas que necesitan ser estudiadas desde un pensamiento holístico y crítico. Así mismo, se expone la posibilidad de considerar las investigaciones desde el enfoque crítico, que según Jones (2010), están centradas en alternativas a los paradigmas investigativos actuales, e implican desarrollar niveles más profundos sobre la problemática. De igual manera, permite trascender la identificación de una problemática universal para plantear la transformación de dichos fenómenos, lo que demuestra un interés de la investigación desde una perspectiva globalizada hasta la especificidad del problema.

Los resultados muestran una tendencia metodológica centrada en la investigación cuantitativa $(91 \%)$ y cualitativa $(9 \%)$. Se justifica esta predominancia a partir de los instrumentos utilizados para establecer la percepción de los estilos de vida, ya que se emplean protocolos estandarizados (Estilo de Vida Fantástico, Pentágono Bienestar y PAR-Q) desde un enfoque cuantitativo.

El grado de concentración de la producción científica, presenta una tendencia del crecimiento de los estudios por países que se relacionan con las revistas indexadas en la temática. En este sentido, el país con más estudios es Brasil (37\%) con la Revista Brasileira de Obesidade, Nutrição e Emagrecimento y la Revista Brasileira em Promoção da Saúde; seguido de España (20\%) con la Revista Retos: Nuevas Tendencias en Educación Física, Deporte y Recreación y la Revista Andaluza de Medicina del Deporte; Colombia (16\%) con la Revista Hacia la Promoción de la Salud y la Revista Cuidarte; México (13\%) con la Revista Salud Pública de México; Portugal (8\%) con la Revista Motricidad; y, por último Argentina (6\%) con la Revista Argentina de Salud Pública. Estos resultados son muy similares con los datos de tendencia investigativa encontrados en el estudio de Delgado-Bravo et al. (2014), donde Brasil presenta un alto grado de concentración de investigaciones en el área de salud, estilo de vida y calidad de vida.

Particularmente en Colombia, los resultados evidencian investigaciones y acciones en el marco de los estilos de vida realizados en conjunto con las universidades. En este sentido, las cinco universidades que más desarrollan estudios en este ámbito son: Universidad Nacional de Colombia (25\%), Universidad de Caldas (23\%), Pontificia Universidad Javeriana (22\%), Universidad de Santander (19\%), y Universidad de Antioquia (11\%). A su vez, esta preocupación con el estilo de vida de los jóvenes universitarios hace parte de las dimensiones de acción del bienestar en las instituciones de educación superior previstas en las políticas establecidas por el Ministerio de Educación Nacional de Colombia (MEN, 2016, p. 28), en las que se requiere de las instituciones desarrollar "[...] estrategias orientadas a la promoción de hábitos saludables y al fomento de la actividad física, el deporte y el adecuado uso del tiempo libre", como también "[...] estrategias relacionadas con la prevención y la promoción de los estilos de vida saludables y del autocuidado".

En el análisis de palabras sobre los artículos sistematizados, se evidencian los principales términos y expresiones utilizadas que están asociadas con las relaciones que se establecen entre estilos de vida y calidad de vida en estudiantes universitarios. En 
este sentido, se utilizó el diagrama radial para exponer de manera ordenada y sistemática las relaciones de palabras según un orden lógico. Por lo anterior, se realizó un análisis lexicométrico identificando las 20 palabras más utilizadas en los estudios, en cuya frecuencia máxima se encuentra «Actividad Física» (518) y con frecuencia mínima los términos «Autocuidado» $\mathrm{y}$ «Antropometría» (37), conforme se presenta en la Figura 3.

Figura 3: Mapa lexicométrico de frecuencia de palabras.

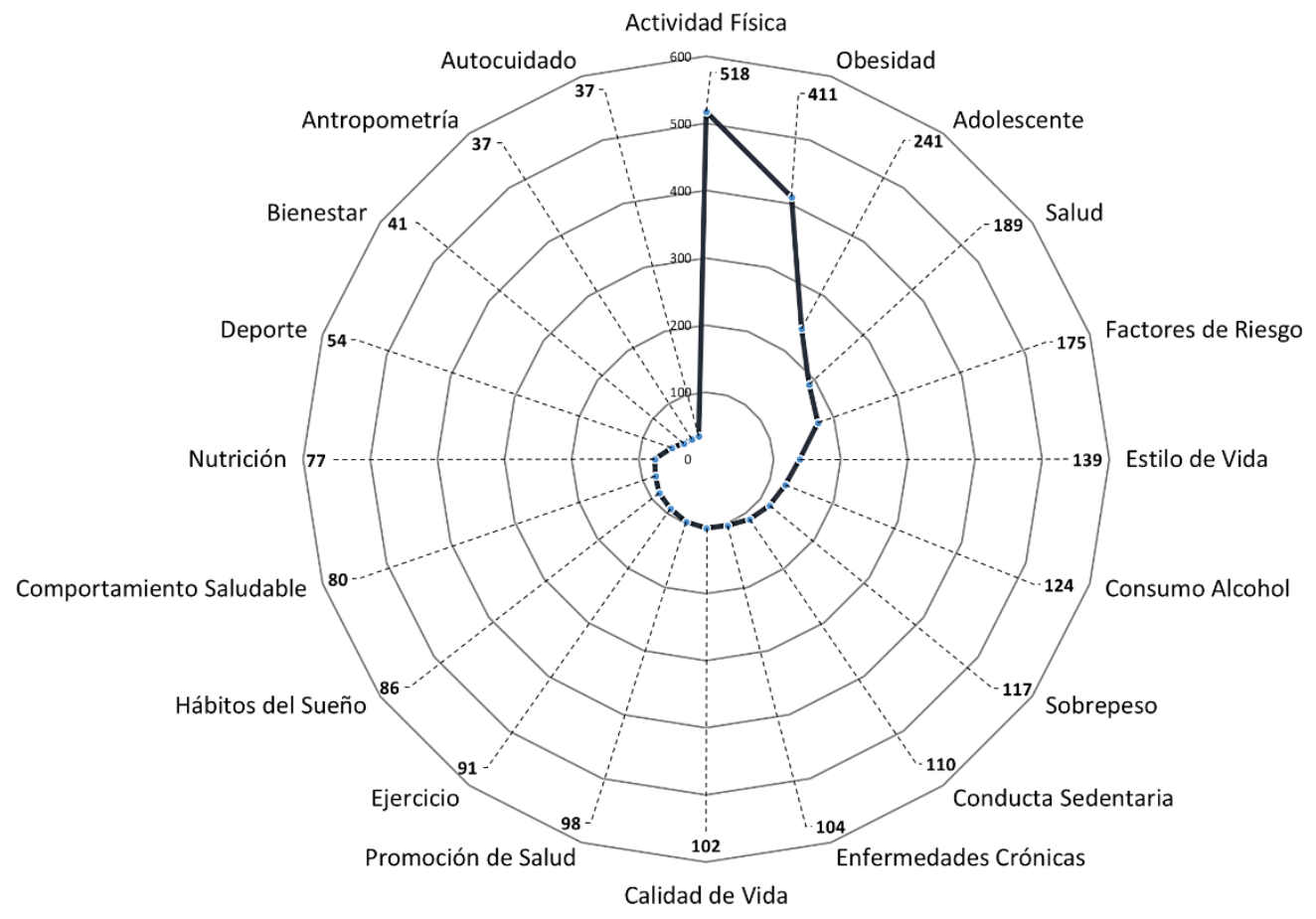

Fuente: Elaboración propia.

A partir del análisis lexicométrico se utilizó el agrupamiento de las palabras en cuatro dimensiones «Actividad Física, Salud, Frecuencia de Consumo y Psicológico» para la interpretación de los resultados y hallazgos desde una perspectiva descriptiva del fenómeno, conforme se presenta en la Figura 4.

Figura 4: Interpretación de los hallazgos.

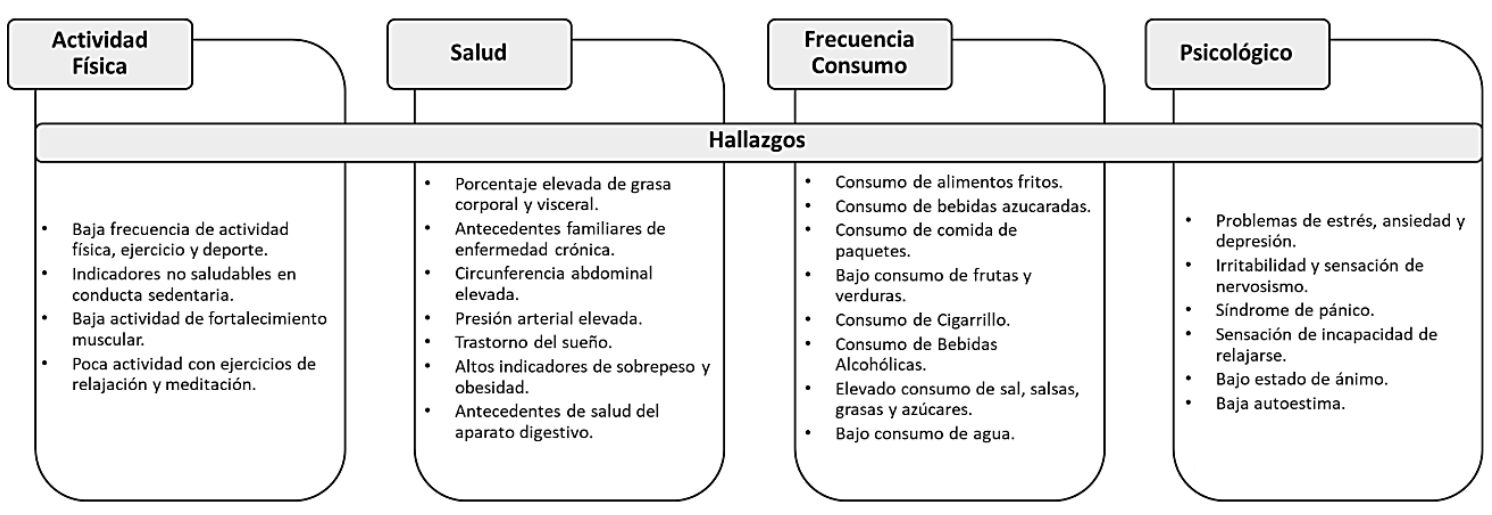

Fuente: Elaboración propia. 
En la Figura 4, se evidencia un panorama nada alentador sobre los estilos de vida en los estudiantes universitarios. Desde la dimensión de «Actividad Física», la problemática está centrada en que los estudiantes no cumplen con las recomendaciones mínimas de actividad física propuestas por la Organización Mundial de la Salud (WHO, 2010) y por el American College of Sports Medicine - ACSM (2018), de un mínimo de 150 minutos de actividad aeróbica de intensidad moderada a la semana, o de 75 minutos de actividad vigorosa; como también, de dos veces a la semana de actividades para el fortalecimiento de los grandes grupos musculares.

Lo anterior, repercute en un estilo de vida no saludable en el ámbito de actividad física, lo cual es propicio para el desarrollo de ECNT. De acuerdo con Booth et al. (2017), la inactividad física y las conductas sedentarias facilitan el aumento y riesgo de sufrir enfermedades, además "[...] es la causa real del desarrollo de 35 enfermedades crónicas, de una menor esperanza de vida y de una mortalidad temprana" (p. 1386). Además, es el origen directo de "[...] acelerar el envejecimiento biológico, pérdidas de aptitud cardiovascular y de fuerza, acortamiento de la vida útil y disminución de la calidad de vida" (BOOTH et al. 2017, p. 1351).

En este sentido, la actividad física está asociada directamente a la segunda dimensión de «Salud», ya que a través de los comportamientos sedentarios y de inactividad física, se desarrolla un aumento del índice de masa corporal, disminución de la elasticidad y movilidad articular, que según la WHO (2017) es la causa del desarrollo de problemas cardíacos, de diabetes y de cáncer del aparato digestivo, además de presentar sensaciones frecuentes de malestar y cansancio físico y mental.

Por lo anterior, se puede afirmar que en el sobrepeso y la obesidad se presenta una acumulación anormal o excesiva de grasa corporal y visceral que puede ser perjudicial para la salud. De acuerdo con la WHO (2017), en el año de 2016, más de 1900 millones de adultos de 18 o más años tenían sobrepeso, de los cuales, más de 650 millones eran obesos. En este sentido, el sobrepeso y la obesidad están vinculados directamente con las muertes por insuficiencia ponderal y uno de los principales problemas de la salud pública actual a nivel mundial.

Por otro lado, este problema presentado desde la perspectiva de la salud, conlleva a su asociación con los hábitos de los estudiantes universitarios, que se puede evidenciar a través de los hallazgos presentados en la dimensión «Frecuencia de Consumo». Sobre todo, se observa que los estudiantes están consumiendo en mayor cantidad alimentos procesados, comidas rápidas, alimentos hipercalóricos, grasas y azucares. Además, en la dieta diaria presentan una baja o insuficiente ingesta de frutas y verduras. Adicional a ello, se evidencia un aumento del consumo de bebidas alcohólicas en el ámbito universitario.

Por último, la dimensión «Psicológico» puede ser considerado un resultado de todas las anteriores dimensiones, ya que el desarrollo de algunas enfermedades puede incidir en el pensamiento, estado de humor y de comportamiento de las personas. Por consiguiente, esta dimensión determina las actividades de manera directa e indirecta relacionadas con el bienestar y que se refleja en la salud mental. De la misma manera, para Papalia, Feldman y Martorell (2012), los factores psicológicos están relacionados con el desarrollo psicosocial y cada etapa de la vida depende de la manera en cómo se enfrenta y se da respuesta a las tareas, ocurrencias y problemas de una manera sana, y así favorecer el desarrollo del ser humano.

Por otro lado, de acuerdo con la Organización Mundial de la Salud - WHO (2015), los factores psicológicos no están relacionados solamente con el desarrollo del ser humano, sino que también están asociados con el deterioro de la salud mental en la población mundial. A su vez, están conexos con los acontecimientos vitales negativos o 
adversidades de una persona, lo que puede afectar directamente su estilo de vida y en consecuencia la salud y la calidad de vida.

Por consiguiente, todas las evidencias presentadas hasta el momento, demuestran un deterioro del estilo de vida en los estudiantes universitarios. Hernando, Oliva y Pertegal (2013) describen la importancia de la adolescencia como momento del ciclo vital para el establecimiento de los hábitos de vida, además es donde se presentan importantes cambios biopsicosociales, en los que los adolescentes realizan sus primeras salidas y reuniones con sus iguales sin control de un adulto y en los que van ganando autonomía para tomar decisiones relacionadas con el ocio y el estilo de vida, lo cual se reflejará en sus hábitos y comportamientos en la vida universitaria.

Desde esta perspectiva, los estilos de vida están asociados a los aprendizajes vivenciados durante el desarrollo de las etapas del ciclo vital humano. En este sentido, el proceso de aprendizaje implica la relación del estilo de vida con el contexto sociocultural en el que se encuentra la persona. Según la teoría ecológica propuesta por Bronfenbrenner (1987), el desarrollo del ser humano ocurre a través de la interacción con el entorno a través de los distintos niveles del ambiente, lo que incide en el estilo de vida de las personas, ya que estos niveles determinan la forma de proceder, pensar y tomar decisiones que fortalecen las relaciones interpersonales dentro del microsistema, mesosistema y exosistema en los que el ser humano está inmerso. A partir de la teoría ecológica, es posible establecer asociaciones e interacciones entre estos sistemas y el desarrollo biopsicosocial y de estilos de vida según su entorno inmediato.

\section{CONSIDERACIONES FINALES}

Los resultados muestran una tendencia de conducta de estilos de vida no saludables en los estudiantes universitarios, lo que a futuro puede generar un deterioro de la salud y calidad de vida. A su vez, los cambios del comportamiento humano están asociados con el entorno y el contexto en que vive. En este sentido, el estilo de vida del ser humano presenta interacciones entre las condiciones de vida y patrones de conductas individuales que son determinados por las características biopsicosociales y por el contexto social y cultural.

En este orden de ideas, en el proceso de satisfacer sus necesidades y en el ejercicio de sus capacidades, el ser humano ha creado diferentes formas de organización, sistemas de pensamiento y acción, cuyo resultado son los diversos contextos sociales y culturales existentes. De esta forma, los estudiantes universitarios son afectados por este contexto social y cultural a partir de los cambios de comportamiento relacionados con el entorno que genera transformaciones en el desarrollo humano y ciclo vital, que inciden en la salud y calidad de vida. Como también, el ciclo vital juega un papel importante en el cambio de los comportamientos de las personas, una vez que se trata de un proceso integrado (biológico, psicológico, social, físico y de salud) que interactúa con el ser humano, donde el ambiente genera transformaciones en los ámbitos del desarrollo y a su vez en los estilos de vida.

Por otro lado, no hay duda de que, en la etapa de la adolescencia, principalmente en el contexto de la "vida universitaria", los estudiantes experimentan nuevas aptitudes sociales y cognitivas; asimismo hábitos, estrategias de afrontamiento, actitudes y valores, que son las bases para consolidar y determinar el desarrollo personal, que presenta repercusiones en la salud y calidad de vida a futuro. Sobre todo, porque el estilo de vida es un comportamiento del ser humano que sufre transformaciones desde 
lo biopsicosocial, lo que propicia cambios de actitudes y conductas a cualquier momento durante el ciclo vital.

A modo de conclusión, partir de esta revisión sistemática, se evidencia la necesidad de realizar más estudios sobre los estilos de vida y los problemas asociados a estos, como reflejo en la calidad de vida desde la perspectiva hacia la promoción de la salud de los estudiantes universitarios. En este sentido, se hace necesario ampliar los estudios del fenómeno desde la perspectiva del desarrollo humano en el marco del ciclo vital, para comprender como los estudiantes universitarios se adaptan a este nuevo paradigma y superan los retos y problemas que se presentan a lo largo de la vida cotidiana universitaria en este mundo globalizado que vivimos.

Para finalizar, se recomienda ampliar las acciones preventivas dirigidas a esta población, a su vez que puedan contribuir al aporte de los Objetivos de Desarrollo Sostenible - ODS, específicamente en el objetivo 3, donde se establece como propósito el de garantizar una vida sana y promover el bienestar de todos a todas las edades; a su vez, con propuestas desde una perspectiva interna de las instituciones de educación superior, como también a través de medidas gubernamentales.

\section{REFERENCIAS}

ACOSTA, C.; JIMÉNEZ, A.; BARBA, C. Expectativas hacia el alcohol en jóvenes universitarios y propuestas comunicacionales. Mediciencias UTA, v. 3, n. 2, p. 33-44, 2019.

ACSM. American College of Sports Medicine. ACSM's guidelines for exercise testing and prescription. Philadelphia: Lipincott Williams \& Wilkins, 2018.

AGUILERA-EGUÍA, R. ¿Revisión sistemática, revisión narrativa o metaanálisis? Revista de la Sociedad Española del Dolor, v. 21, n. 6, p. 359-360, 2014.

ALONSO, A.; MARTÍNEZ, A.; BANDA, F. Estilos de vida saludables en la formación integral del estudiante universitario. Revista Didasc@ lia: Didáctica y Educación, v. 10, n. 2, p. 79-88, 2019.

ÁLVAREZ, J.; HARO, G. Millennials: la generación emprendedora. Madrid: Editorial Ariel S.A., 2017.

ARESTEDT, L.; PERSSON, C.; BENZEIN, E. Living as a family in the midst of chronic illness. Scand J Caring Sci, v. 28, n. 1, p. 29-37, 2014.

BLOOMBERG, L. D.; VOLPE, M. F. Completing your qualitative dissertation: a road map from beginning to end. London: Sage, 2016.

BOOTH, F.; ROBERTS, C. K.; THYFAULT, J. P.; RUEGSEGGER, G. Role of inactivity in chronic diseases: evolutionary insight and pathophysiological mechanisms. Physiological Reviews, v. 97, p. 1351-1402, 2017.

BRAVO-AVALOS, M. B.; LÓPEZ-SALAZAR, J. L.; VELASCO-MATVEEV, L. A.; MIRANDA, M. F. Comercialización, consumo y valor nutricional de la comida rápida, en los estudiantes universitarios, caso: carrera de agroindustria de la facultad de ciencias pecuaria. KnE Engineering, v. 5, n. 2, p. 94-106, 2020.

BRONFENBRENNER, U. La ecología del desarrollo humano: experimentos en entornos naturales y diseñados. Barcelona: Ediciones Paidós, 1987.

CARRILLO, A. Globalización: revolución industrial y sociedad de la información. Revista Ciencia, v. 19, n. 2, p. 269-284, 2017.

CENTERS FOR DISEASE CONTROL AND PREVENTION. Strategies to improve the quality of physical education. USA: Department of Health and Human Services, 2018. 
COTRINA, J.; RODRÍGUEZ, J. Estilo de vida y factores biosocioculturales del adulto joven. H.U.P. Lomas del Sur, Nuevo Chimbote. In Crescendo, v. 5, n. 1, p. 13-22, 2014.

DA COSTA, B. G. G.; DA SIlVA, K. S.; DA SILVA, J. A.; MINATTO, G.; LIMA, L. R. A.; PETROSKI, E. L. Sociodemographic, biological, and psychosocial correlates of light- and moderate-tovigorous-intensity physical activity during school time, recesses, and physical education classes. Journal of Sport and Health Science, v.8, p. 177-182, 2019.

DE SOUZA MARTINS, M.; ZEA ROBLES, C.; RODRIGUEZ CARO, G. C.; MOLINA PINZÓN, A. Estilo de vida y factores socioeconómicos en estudiantes de electivas de actividad física y deporte de la Pontificia Universidad Javeriana. Análisis, v. 49, n. 90, p. 229-243, 2017.

DE SOUZA MARTINS, M.; MOLINA PINZON, A.; ZEA ROBLES, A. C. Analysis of the Lifestyle of Students of Pontificia Javeriana University Colombia. Medicine \& Science in Sports \& Exercise, v. 47, n. 5, p. 915, 2015.

DELGADO-BRAVO, A. I.; Naranjo-Toro, M.; Castillo, R.; Basante, Y.; Rosero-Otero, M. Tendencias de investigación en salud: análisis y reflexiones. Aquichan, v. 14, n. 2, p. 237-250, 2014.

GALINDO, L. Experiencias de formación en investigación de los maestros de Cundinamarca. Bogotá: Centro Editorial UNIMINUTO, 2016.

GÓMEZ-ACOSTA, C. Factores psicológicos predictores de estilos de vida saludable. Revista de Salud Pública, v. 20, n. 2, p. 155-162, 2018.

GONZÁLEZ-PÁRAMO, J. Cuarta revolución industrial, empleo y estado del bienestar. Madrid: Real Academia de la Ciencias Morales y Políticas, 2017.

GRAU, J. Enfermedades crónicas no trasmisibles un abordaje desde los factores psicosociales. Salud \& Sociedad, v. 7, n. 2, p. 138-166, 2016.

HARRIS, A. Report, finding our own way: mental health and moving from school to further and higher education. London: Centre For Mental Health, 2019.

HERNÁNDEZ SAMPIERI, R.; FERNÁNDEZ COLLADO, C.; BAPTISTA LUCIO, M. P. Metodología de la investigación. México: Mc Graw Hill Education, 2014.

HERNANDO, A.; OLIVA, A.; PERTEGAL, M. Diferencias de género en los estilos de vida de los adolescentes. Psychosocial Intervention, v. 22, p. 15-23, 2013.

JONES, K. The practice of quantitative methods, chapter 23. In SOMEKH, B.; LEWIN C. (eds.). Research methods in the social sciences. Newbury Park: SAGE Publications Ltd, 2010. p. 201-211.

KHAN, K. S.; KUNZ, R.; KLEIJNEN, J.; ANTES, G. Five steps to conducting a systematic review. Journal of the Royal Society of Medicine, v. 96, p. 118-121, 2003.

KRUPP, M.; CHATTON, M. Current medical diagnosis \& treatment. Los Altos: Lange Medical, 2014.

MEN. Lineamientos de política de bienestar para instituciones de educación superior. Bogotá, Colombia: MEN, 2016.

MOHER, D.; LIBERATI, A.; TETZLAFF, J.; ALTMAN, D. G.; PRISMA Group. Preferred reporting items for systematic reviews and metaanalyses: The PRISMA statement. Annals of Internal Medicine, v. 51, n. 4, p. 264-272, 2009.

NAHAS, M. Atividade física, saúde e qualidade de vida: conceitos e sugestões para um estilo de vida ativo. Londrina: Midiograf, 2013. 
PAPALIA, D. E.; FELDMAN, R. D.; MARTORELL, G. Desarrollo humano. Bogotá: McGraw-Hill Education, 2012.

PEW RESEARCH CENTER. Millennials: a portrait of generation next. Confident. Connected. Open to Change. feb. 2010. Disponible en: https://www.pewresearch.org/wpcontent/uploads/sites/3/2010/10/millennials-confident-connected-open-to-change.pdf. Acceso en: 19 sep. 2020.

QUILIANO, M.; QUILIANO, M. Inteligencia emocional y estrés académico en estudiantes de enfermería. Revista Ciencia y Enfermería, v. 26, n. 3, p. 1-9, 2020.

ROJAS OSPINA, P.; DÍAZ RINCÓN, M.; DE SOUZA MARTINS, M.; POSADA-BERNAL, S. Lifestyles and academic achievement in students of children's education program of the Pontificia Universidad Javeriana - Colombia. EDULEARN18 Proceedings, p. 1751-1758, 2018.

VIDARTE-CLAROS, J. A.; VÉLEZ-ÁLVAREZ, C.; PARRA-SÁNCHEZ, J. H. Niveles de sedentarismo en población de 18 a 60 años. Revista de Salud Pública, v. 14, n. 3, p. 417-428, 2012.

WHO. Global Health Risk-Mortality and burden of disease attributable to selected major risk Cancer. Ginebra: WHO, 2017.

WHO. World Health Organization. Evaluación de necesidades y recursos psicosociales y de salud mental: guía de herramientas para contextos humanitarios. Ginebra: WHO, 2015.

WHO. World Health Organization. Action plan for the global strategy for the preventión and control of noncommunicable diseases. Ginebra: WHO, 2013.

WHO. World Health Organization. Global recommendations on physical activity for health. Ginebra: WHO, 2010

WHO. World Health Organization. Un enfoque de la salud que abarca la totalidad del ciclo vital: repercusiones para la capacitación. Ginebra:WHO, 2000.

Recibido en: 26 set. 2020.

Aprobado en: 25 nov. 2020. 